\title{
HD 161306: a radiatively interacting Be binary? ${ }^{\star}, \star \star$ (Research Note)
}

\author{
P. Koubský ${ }^{1}$, L. Kotková ${ }^{1}$, M. Kraus ${ }^{1}$, S. Yang ${ }^{2}$, M. Šlechta ${ }^{1}$, P. Harmanec ${ }^{4}$, M. Wolf ${ }^{4}$, V. Votruba ${ }^{1,3}$, J. Kubát ${ }^{1}$, \\ B. Kubátová ${ }^{1}$, E. Niemczura ${ }^{5}$, and P. Škoda ${ }^{1}$ \\ 1 Astronomical Institute, Academy of Sciences of the Czech Republic, Fričova 298, 25165 Ondřejov, Czech Republic \\ e-mail: koubsky@sunstel.asu.cas.cz \\ 2 Department of Physics and Astronomy, University of Victoria, Victoria BC V8W 3P6, Canada \\ 3 Department of Theoretical Physics and Astrophysics, Masaryk University, Kotlářská 2, 61137 Brno, Czech Republic \\ 4 Astronomical Institute of the Charles University, Faculty of Mathemathics and Physics, V Holešovičkách 2, 18000 Praha 8, \\ Czech Republic \\ 5 University of Wrocław, Astronomical Institute, Kopernika 11, 51-622 Wrocław, Poland
}

Received 17 April 2014 / Accepted 29 May 2014

\section{ABSTRACT}

\begin{abstract}
The spectrum of the Be star HD 161306 is shown to vary periodically with a period of $\sim 100$ days. The radial velocity of the He I $6678 \AA$ emission peak varying in antiphase to the radial velocity of the $\mathrm{H} \alpha$ emission wings component suggests that the star is a binary similar to $\phi$ Per, 59 Cyg, or FY CMa, i.e. a radiatively interacting Be binary - a rare case among Be stars. This type of object is also called a $\phi$ Per-type binary or $\mathrm{Be}+\mathrm{sdO}$ binaries. The range of radial-velocity variations of the strong emission peak in the helium line observed in HD 161306 is about $180 \mathrm{~km} \mathrm{~s}^{-1}$, similar to what is observed for these systems. We therefore conclude that HD 161306 may represent another case of a Be star with a hot subdwarf companion.
\end{abstract}

Key words. binaries: spectroscopic - stars: emission-line, Be - stars: individual: HD 161306

\section{Introduction}

The star HD 161306 (MWC 271, HIP 86884, SAO 141851) is a relatively bright $\mathrm{Be} \operatorname{star}\left(V=8^{\mathrm{m}} \cdot 30, \mathrm{~B} 0:\right.$ ne $\left.\mathrm{C}\right)$. The $\mathrm{H} \alpha$ emission in HD 161306 was first observed by Humason on August 1, 1922 (Humason \& Merrill 1922). The target is listed in the first edition of the MWC catalogue (Merrill \& Burwell 1933, see also references therein). HIPPARCos data show photometric long-term variation with an amplitude of $0^{\mathrm{m}} .1$ during the active lifetime of the satellite. In August 2012, HD 161306 was put on the observing list of Be stars of the Ondřejov $2 \mathrm{~m}$ Telescope. A changing pattern in the helium line He I $6678 \AA$ was readily visible at early inspection of the data: an emission component swaying from the red side of the profile to the blue and back. Since June 2013, HD 161306 has also been observed with the 1.22-m Telescope at the Dominion Astrophysical Observatory (DAO) in Victoria, Canada. In this study we present preliminary results of the analysis of the emission in $\mathrm{H} \alpha$ and of He I $6678 \AA$ lines in the spectra of HD 161306.

* Based on observations obtained with the Perek 2-m Telescope at Ondřejov Observatory, Czech Republic, and with the 1.22-m Telescope of the Dominion Astrophysical Observatory, Canada. This work made use of the BeSS database, operated at LESIA, Observatoire de Meudon, France.

$\star \star$ The spectra taken in Ondřejov ( $\mathrm{H} \alpha$ and helium regions) are only available in electronic form (FITS format) at the CDS via anonymous ftp to cdsarc.u-strasbg. fr (130.79.128.5) or via http://cdsarc.u-strasbg.fr/viz-bin/qcat?]/A+A/567/A57

\section{Spectroscopic observations and their reductions}

Most of the spectra of HD 161306 used in this study were obtained the with the Ondřejov single-order spectrograph + Pylon $2048 \times 512$, which has replaced the old SITe $2000 \times 800$ chip in the focus of a $702 \mathrm{~mm}$ camera $(R=12000$, ranges $6250-6750$ and 8392-8900 $)$ ). The spectrograph was attached to the coudé focus of the P2MT telescope. In the case of the DAO observations, spectra were obtained with the McKellar Spectrograph in the coudé focus of the $1.22 \mathrm{~m}$ telescope, SITe-4 $4096 \times$ 2048 chip, camera/grating configuration $32121 \mathrm{H}(R=18000$, range 6330-6940 $\AA$ ). Most of the exposures at DAO were secured in robotic mode. In all cases, calibration arc frames were taken before and after each stellar frame. During each night, a series of flat field and bias exposures were obtained. The stellar and calibration spectra (Th-Ar and tungsten lamps) were reduced using IRAF. Altogether, one infrared and forty three red spectra were secured. In addition, we downloaded spectra of HD 161306 from the BeSS database. However, the quality of most of them (five out of six) was poor, so only one BeSS spectrum was used. It was obtained by Joan Garro Fló with the LHires-B spectrograph $(R=5000)$ attached to a MEADE SC16 telescope located at Piera near Barcelona, Spain.

Details describing the reduction of the spectrum can be found in the BeSS database. In all cases, the heliocentric corrections were applied. The spectra were rectified to a unit continuum, and radial velocities (RVs) were measured with the help of the SPEFO code written by Dr. Horn and more recently improved by P.Š. and Mr. Krpata (see Horn et al. 1996; Škoda 1996). The zero point of the RV scale was corrected using reliable telluric lines. The stellar RVs were measured interactively, 

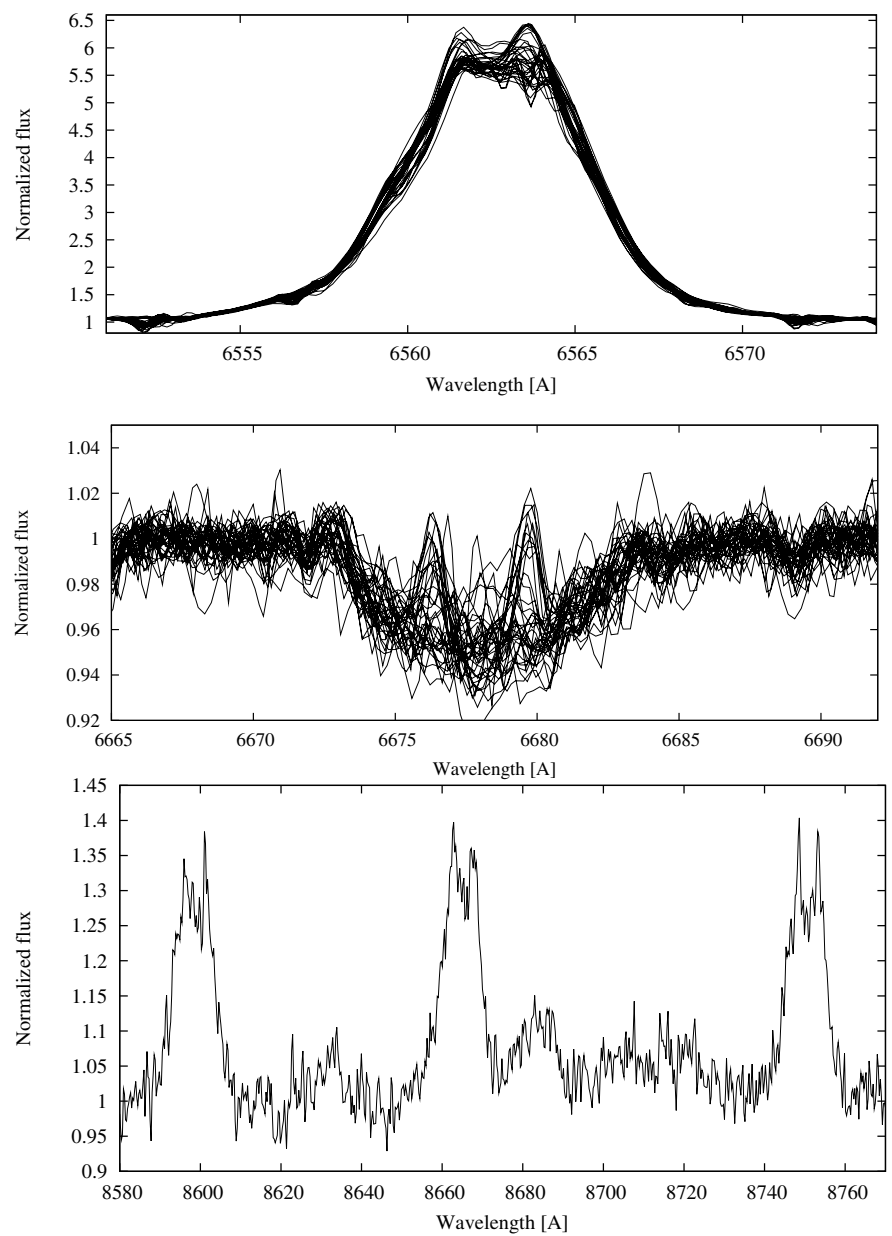

Fig. 1. Available spectra of HD 161306: H $\alpha$ - top panel; He I $6678 \AA$ middle panel; Paschen lines - bottom panel.

comparing the direct and flipped images of the profile seen on a computer screen. This way, one can measure the position of the chosen part of the profile. The SPEFO code transforms the position to the wavelength using the dispersion curve defined by the positions of selected unblended lines of the comparison spectra. The wavelengths are then transformed to RVs using the particular laboratory wavelength of measured line. As we did for some other Be stars, we focused on the steep lower wings of $\mathrm{H} \alpha$, and the strong emission component of the He I $6678 \AA$ line. This method works very well for objects with symmetrical profiles (see e.g. Božić et al. 1995; Koubský et al. 2010; Nemravová et al. 2012; Peters et al. 2013).

The basic reasons for the RV curve of the strong $\mathrm{H} \alpha$ emission wings giving the most reliable estimate of the true orbital motion of the Be primary can be found in Ruždjak et al. (2009) or Harmanec et al. (2002). The errors of the method depend on the signal-to-noise ratio $(\mathrm{S} / \mathrm{N})$ of the particular spectrum and on the shape of the profile. In the case of asymmetric profiles, we set the coincidence of the two images at the 0.5 level of the maximum intensity. The profiles used for RV measurements are shown in Fig. 1 (top and middle panels). A part of the infrared spectrum displaying double-peaked Paschen lines in emission is shown in the bottom panel of Fig. 1. Table 1 lists the heliocentric Julian dates, phases (for the ephemeris see Sect. 3), along with the radial velocities for the emission of $\mathrm{H} \alpha$ line, the main emission component of the helium line He I $6678 \AA$, and the origin of
Table 1. Radial velocity measurements.

\begin{tabular}{|c|c|c|c|c|}
\hline $\begin{array}{l}\text { Date } \\
\text { (HJD-2 } 400 \text { 000) }\end{array}$ & Phase & $\begin{array}{r}\mathrm{H} \alpha \\
\mathrm{km} \mathrm{s}^{-1}\end{array}$ & $\begin{array}{r}\text { He I } 6678 \AA ̊ \\
\mathrm{~km} \mathrm{~s}^{-1}\end{array}$ & Source \\
\hline 56162.3004 & 0.101 & $3.1 \pm 6.1$ & $-66.8 \pm 2.8$ & OND \\
\hline 56418.5474 & 0.665 & $-10.1 \pm 7.2$ & $30.3 \pm 5.0$ & OND \\
\hline 56446.9480 & 0.950 & $-0.7 \pm .5 .0$ & $-93.2 \pm 3.1$ & DAO \\
\hline 56455.4935 & 0.035 & $-4.3 \pm 6.6$ & $-83.5 \pm 4.5$ & OND \\
\hline 56478.4464 & 0.265 & $-11.9 \pm 7.0$ & $16.0 \pm 5.2$ & OND \\
\hline 56481.4163 & 0.294 & $-12.3 \pm 8.2$ & $22.1 \pm 6.1$ & OND \\
\hline 56490.4939 & 0.385 & $-13.3 \pm 5.2$ & $63.2 \pm 4.7$ & OND \\
\hline 56497.4763 & 0.455 & $-12.5 \pm 5.8$ & $70.3 \pm 4.1$ & OND \\
\hline 56499.4277 & 0.475 & $-13.1 \pm 7.0$ & $68.9 \pm 6.1$ & OND \\
\hline 56504.383 & 0.524 & $-20.1 \pm 4.8$ & $65.3 \pm 5.9$ & BeSS \\
\hline 56506.3534 & 0.544 & $-11.8 \pm 6.9$ & $71.3 \pm 4.3$ & OND \\
\hline 56510.3872 & 0.584 & $-11.8 \pm 8.1$ & $75.8 \pm 3.8$ & OND \\
\hline 56514.7118 & 0.623 & $-15.2 \pm 6.2$ & $65.6 \pm 4.1$ & DAO \\
\hline 56517.7591 & 0.658 & $-13.8 \pm 6.3$ & $65.3 \pm 3.4$ & DAO \\
\hline 56519.3786 & 0.674 & $-14.9 \pm 6.0$ & $63.4 \pm 3.7$ & OND \\
\hline 56535.6909 & 0.838 & $-6.4 \pm 8.0$ & n.v. & DAO \\
\hline 56541.3339 & 0.894 & $-2.1 \pm 7.5$ & $-86.4 \pm 2.8$ & OND \\
\hline 56545.3031 & 0.934 & $-10.8 \pm 5.0$ & $-91.8 \pm 4.0$ & OND \\
\hline 56550.2704 & 0.984 & $-4.6 \pm 9.5$ & $-87.0 \pm 4.3$ & OND \\
\hline 56555.6476 & 0.037 & $-4.4 \pm 7.0$ & $-84.1 \pm 3.8$ & DAO \\
\hline 56558.6668 & 0.068 & $-5.2 \pm 7.2$ & $-77.4 \pm 3.9$ & DAO \\
\hline 56560.2894 & 0.084 & $-3.1 \pm 5.0$ & $-80.6 \pm 4.6$ & OND \\
\hline 56563.3202 & 0.114 & $-6.8 \pm 4.0$ & $-94.3 \pm 3.9$ & OND \\
\hline 56566.2616 & 0.144 & $-2.6 \pm 6.8$ & $-72.9 \pm 4.0$ & OND \\
\hline 56569.6240 & 0.177 & $-10.8 \pm 7.2$ & $-36.7 \pm 5.0$ & DAO \\
\hline 56572.2657 & 0.204 & $-10.0 \pm 7.5$ & $-38.1 \pm 4.0$ & OND \\
\hline 56574.2393 & 0.223 & $-4.9 \pm 9.3$ & $-10.7 \pm 6.1$ & OND \\
\hline 56594.2007 & 0.423 & $-13.7 \pm 6.3$ & $67.7 \pm 4.0$ & OND \\
\hline 56597.2078 & 0.453 & $-17.5 \pm 7.4$ & $62.8 \pm 4.9$ & OND \\
\hline 56604.1980 & 0.523 & $-18.1 \pm 8.1$ & $72.3 \pm 3.9$ & OND \\
\hline 56713.6671 & 0.619 & $-19.2 \pm 5.9$ & $64.1 \pm 5.4$ & OND \\
\hline 56727.6800 & 0.759 & $-9.8 \pm 6.1$ & $37.8 \pm 8.9$ & OND \\
\hline 56728.6664 & 0.769 & $-12.8 \pm 7.8$ & $-47.4 \pm 9.4$ & OND \\
\hline 56729.6668 & 0.779 & $-10.3 \pm 5.9$ & n.v. & OND \\
\hline 56730.6634 & 0.789 & $-11.9 \pm 4.9$ & $-77.3 \pm 4.8$ & OND \\
\hline 56736.6652 & 0.849 & $-8.0 \pm 5.2$ & n.v. & OND \\
\hline 56737.5953 & 0.858 & $-7.4 \pm 3.8$ & n.v. & OND \\
\hline 56744.6200 & 0.928 & $-8.0 \pm 5.2$ & $-89.2 \pm 4.8$ & OND \\
\hline 56745.6035 & 0.938 & $-7.2 \pm 3.2$ & $-89.5 \pm 3.3$ & OND \\
\hline 56748.6075 & 0.968 & $-6.5 \pm 6.1$ & n.v. & OND \\
\hline 56750.6363 & 0.989 & $-6.7 \pm 5.2$ & $-88.5 \pm 2.8$ & OND \\
\hline 56754.6048 & 0.028 & $-5.8 \pm 4.9$ & $-92.7 \pm 2.8$ & OND \\
\hline 56755.5930 & 0.038 & $-5.1 \pm 6.2$ & $-89.1 \pm 4.7$ & OND \\
\hline
\end{tabular}

Notes. Column 3 - n.v. means that emission component is not visible; column 5 - Source explains the origin of spectra Ondřejov - OND; Dominion Astrophysical Observarory - DAO; BeSS database - BeSS. The first two Ondřejov spectra were recorded with the SITe chip, the rest with the Pylon chip.

spectra. The errors were determined from the repeated measurements made by members of the team.

\section{Data analysis}

Inspection of Table 1 clearly shows that the radial velocity of the main emission component in the helium line He I $6678 \AA$ varies with time - see also the profiles in the middle panel in Fig. 1. First we tried to estimate the possible periodicity of the RVs with Stellingwerf's (1978) phase dispersion minimization (PDM) method (38 points) in the range of frequencies $0.001-0.1 \mathrm{~cd}^{-1}$. The PDM search indicated the best period $P=99.20635$ days with $\theta=0.0576$. The period was found to 
Table 2. Formal orbital elements for HD 161306.

\begin{tabular}{llc}
\hline \hline Solution & Circular & Eccentric \\
\hline$P(\mathrm{~d})$ & $99.90 \pm 0.50$ & $100.03 \pm 0.48$ \\
$T_{\operatorname{maxRV}}$ & $2456152.34 \pm 2.16$ & $2456148.5 \pm 10.3$ \\
$e$ & 0.0, assumed & $0.106 \pm 0.033$ \\
$\omega(\mathrm{deg})$ & - & $269.8 \pm 17.3$ \\
$K_{\mathrm{H} \alpha}\left(\mathrm{km} \mathrm{s}^{-1}\right)$ & $4.90 \pm 1.53$ & $5.03 \pm 1.64$ \\
$K_{\mathrm{HeI}}\left(\mathrm{km} \mathrm{s}^{-1}\right)$ & $86.48 \pm 2.46$ & $87.43 \pm 2.50$ \\
$\gamma\left(\mathrm{km} \mathrm{s}^{-1}\right)$ & $-8.32 \pm 1.29$ & $9.00 \pm 1.25$ \\
$\mathrm{rms}\left(\mathrm{km} \mathrm{s}^{-1}\right)$ & 11.363 & 10.801 \\
Number of spectra & $43 / 39$ & $43 / 39$ \\
\hline
\end{tabular}

Notes. Component $1-\mathrm{RV}$ of $\mathrm{H} \alpha$ emission line; component $2-\mathrm{RV}$ of prominent emission peak in the He I $6678 \AA$ line.

be significant with $p<0.01$ using the test described by Linnell Nemec \& Nemec (1985). We used the value of 99 days as an input parameter to the code SPEL (written by Dr. Horn and never published) designed for orbital solutions of spectroscopic binaries to derive RV curves based both on $\mathrm{H} \alpha$ emission lines (component 1) and maximum helium emission peak (component 2).

In the first step we allowed for a circular orbit and converged period $P$, the time of maximum radial velocity $T_{\operatorname{maxRV}}$, semiamplitudes $K_{\mathrm{H} \alpha}, K_{\mathrm{He}}$, and the value of the systemic or $\gamma$ velocity. In the second step we also allowed for an elliptical-orbit solution, adding the eccentricity $e$ and longitude of the periastron $\omega$. Table 2 summarizes the elements for the solutions with code SPEL and the rms errors. The orbital solutions were not aimed at deriving the true orbital elements of the binary since the emission peak in the He I line need not be centred on the centre of the gravity of the secondary. Their purpose was to improve the value of the orbital period using more data $(39+43$ points $)$ within one program run. The eccentric solution yielded a better fit, and also the errors of some elements are lower. Given the value of $\omega$ close to $270^{\circ}$, the eliptical orbit is suspect; however, since it might be caused by the distortion of the material responsible for the emission, which is almost filling the corresponding Roche lobe (see, e.g., Harmanec 2002). For the moment, we therefore have adopted the period from the circular solution $-P=99.90 \pm 0.50$, and use it in all phase diagrams presented there. Phase 0.0 corresponds to the time of $\mathrm{RV}_{\max }-\mathrm{JD}=56152.24 \pm 2.19$. Figure 2 shows a montage of all He I $6678 \AA$ line profiles as a grey-scale image, and Fig. 3 displays velocities from Table 1.

\section{Discussion}

Our work demonstrates that the spectroscopic variability of HD 161306 is dominated by a period of $\sim 100$ days. The phase variation of the principal emission component of He I $6678 \AA$ with this period is presented in Fig. 2 (top panel), while in Fig. 3 we show RV curves for the helium emission component and for $\mathrm{H} \alpha$ emission folded with the same period. Since the emission component is strongest at velocity extremes and weaker when at zero velocity, it seems that similar to the case of $o$ Pup (see Koubsky et al. 2012), the strength of the He I $6678 \AA$ A emission feature depends on phase. Connection with other $\phi$ Per-like binaries has already been discussed in Koubsky et al. (2012). In Table 3 we give a comparison of basic properties of $\phi$ Per-type binaries with those of HD 161306. The spectral types of the confirmed $\mathrm{Be}+\mathrm{sdO}$ binaries are within the range $\mathrm{B} 0-\mathrm{B} 2$ and luminosity class $\mathrm{V}$.

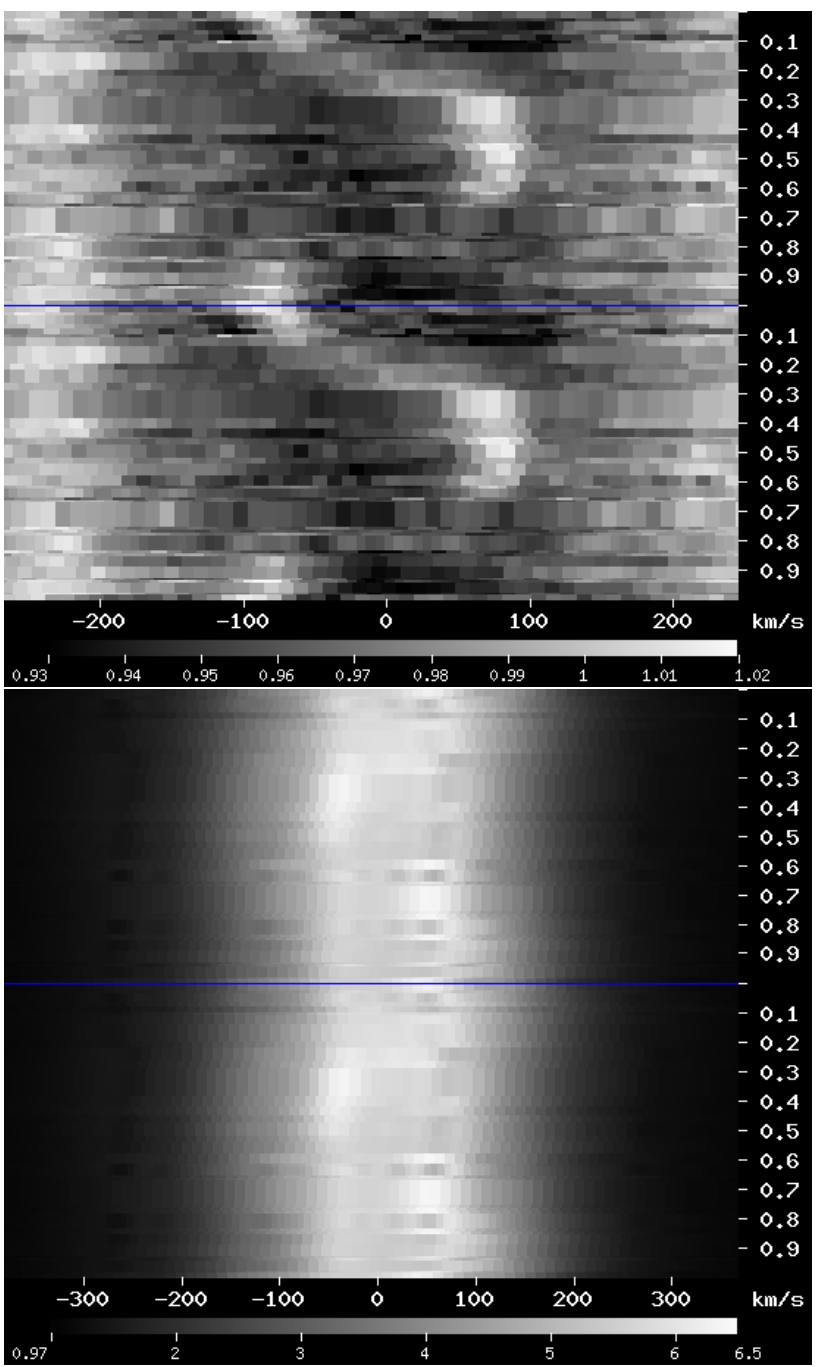

Fig. 2. Trailed spectra of HD 161306 arranged according to the phase of the period $99.90^{d}$ in a grey-scale representation. Top panel: He I $6678 \AA$ line, bottom panel: $\mathrm{H} \alpha$ line. The spectral image for the orbital cycle is reproduced twice to improve the sense of phase continuity. Also, different velocity widths are used in the upper and lower panels.

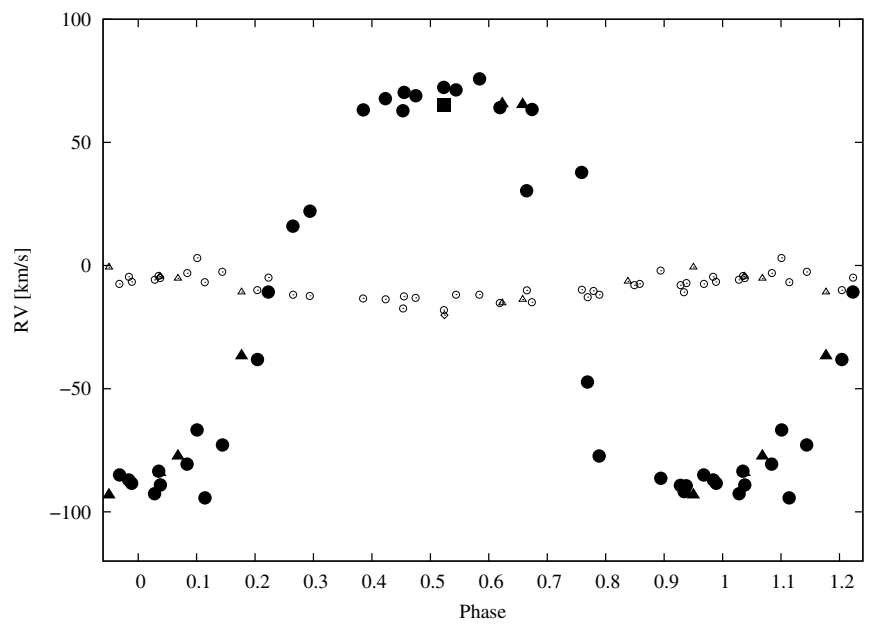

Fig. 3. Radial velocity of the prominent emission peak in the profile of He I $6678 \AA$ folded with the 99.90 -day period represented by solid symbols: dots Ondřejov, triangles DAO, square BeSS. The open symbols indicate the emission velocities of $\mathrm{H} \alpha$ : circles Ondřejov, triangles DAO, diamond BeSS. 
Table 3. Comparison of HD 161306 with the confirmed Be binaries showing the effect of radiative interaction.

\begin{tabular}{llcccccccl}
\hline \hline Star & Sp. type & $\begin{array}{c}v \sin i \\
\mathrm{~km} \mathrm{~s}^{-1}\end{array}$ & $\begin{array}{c}I_{\mathrm{c}} \mathrm{H} \alpha \\
F / F_{\mathrm{c}}\end{array}$ & $\begin{array}{c}F W H M \mathrm{H} \alpha \\
\AA\end{array}$ & $\begin{array}{c}\text { Orbital per. } \\
\mathrm{d}\end{array}$ & $\begin{array}{c}K_{1} \\
\mathrm{~km} \mathrm{~s}^{-1}\end{array}$ & $\begin{array}{c}K_{2} \\
\mathrm{~km} \mathrm{~s}^{-1}\end{array}$ & $\begin{array}{c}\text { Range } \\
\mathrm{km} \mathrm{s}^{-1}\end{array}$ & Reference \\
\hline$\phi$ Per & B2pe & 450 & 4.0 & 9 & 126.673 & 9.97 & 81.3 & \pm 120 & Gies et al. (1998) \\
FY CMa & B0.5IVe: & 340 & 1.8 & 11 & 37.253 & $17.4(14.4)$ & 128.2 & $\pm 185:$ & Peters et al. (2008) \\
59 Cyg & B1-1.5Ve & 450 & 2.0 & 12 & 28.187 & 11.7 & 121.3 & \pm 180 & $\begin{array}{l}\text { Peters et al. (2013) } \\
\text { HD 161306 }\end{array}$ \\
B0:ne & $160:$ & 6.2 & 7 & 99.90 & $5:$ & & \pm 87 & This work \\
\hline
\end{tabular}

The spectral type of HD 161306 is taken from the SIMBAD Astronomical Database, since the only line suitable for determining the effective temperature and luminosity of HD 161306 available to us is the He I $6678 \AA$ line influenced by the variable emission component. Reliable determination of the spectral type and luminosity of HD 161306 should be based on lines in the blue part of the spectrum. We intend to focus on this issue in future studies of this star. The difference in rotational velocity and range of RV curve for the helium He I $6678 \AA$ emission feature seems to be the consequence of lower inclination of the rotational axis of the Be star and the disk. A more pole-on orientation of the system might also be supported by the $\mathrm{H} \alpha$ line, which is narrower but stronger than in the other systems listed in Table 3.

Although we lack any direct evidence for lines of the hot subdwarf and the RV curve of the primary star presented in Fig. 3 shows an amplitude only three times exceeding its error, we suggest that HD 161306 is a new strong candidate for a $\mathrm{Be}+\mathrm{sdO}$ binary. The velocity curve of $\mathrm{H} \alpha$ emission can be discerned in Fig. 2 (bottom panel), as well as the $V / R$ variation in $\mathrm{H} \alpha$ emission line $(V>R$ near phase $0.3, V<R$ near phase 0.8 ). New phase-resolved observations of HD 161306 covering a broader spectral interval with higher $\mathrm{S} / \mathrm{N}$ are needed to (i) bring direct evidence of the hot subdwarf and derive reliable orbital parameters for both components; and (ii) to map the behaviour of the hot spot in the disk. To monitor the infrared spectral range might be particularly important. Since the Paschen lines are all in emission (see Fig. 1, bottom panel), their profiles might be more valuable tracers for the intrinsic kinematics of the primary than the $\mathrm{H} \alpha$ line, because their radial velocity can be more easily measured.

A search for other $\phi$ Per-type binaries (three stars listed in Table 3 are very bright objects) is very tempting. However, the example of HD 161306 shows that the excess emission from the outer part of the Be star disk can be quite tiny, and its profile fairly complicated. In this context, one should think about systematic phase-resolved observations of helium lines in HR 2142
- a Be star that has been on the list of candidates for radiatively interacting Be binaries for more than a decade (Peters \& Gies 2002). A larger sample of $\phi$ Per-type binaries would offer us the unique opportunity to study both the outcome of close binary processes at a critical point of evolutionary sequences and the structure of Be star disks.

Acknowledgements. This research was conducted in part with funding by the ESA PECS 98058 contract to the Astronomical Institute of the Academy of Sciences of the Czech Republic, and by LG14032 of MŠMT ČR. M.K. acknowledges financial support from GAČR (grant number 14-21373S). P.H. and M.W. were supported by the GAČR grant P209/10/0715. E.N. acknowledges the support from 1007/S/IAs/14 funds and funding through NCN grant 2011/01/B/ST9/05448. The Astronomical Institute of the Academy of Sciences of the Czech Republic is supported by project RVO:67985815. We acknowledge the use of the only one good spectrum from the BeSS database. Most of the data obtained at DAO were secured in the robotic mode of the 1.22-m telescope. It is a pleasure to thank Dr. D. Bohlender, one of the key people of this highly useful and successful project. We thank Mr. J. Čechura, who at our request obtained some of the Ondřejov spectra used in this study.

\section{References}

Božić, H., Harmanec, P., Horn, J., et al. 1995, A\&A, 304, 235

Gies, D. R., Bagnuolo, Jr., W. G., Ferrara, E. C., et al. 1998, ApJ, 493, 440

Harmanec, P. 2003, in Çannakkale Astrophysics Workshop 2002, eds. O. Demircan \& E. Budding, 221

Harmanec, P., Božić, H., Percy, J. R., et al. 2002, A\&A, 387, 580

Horn, J., Kubát, J., Harmanec, P., et al. 1996, A\&A, 309, 521

Humason, M. L., \& Merrill, P. V. 1922, PASP, 34, 351

Koubský, P., Hummel, C. A., Harmanec, P., et al. 2010, A\&A, 517, A24

Koubský, P. Kotková, L., Šlechta, M., et al. 2012, A\&A, 545, A121

Linnell Nemec, A. F., \& Nemec, J. M. 1985, AJ, 90, 2317

Merrill, P. W., \& Burwell, C. G. 1933, ApJ, 78, 87

Nemravová, J., Harmanec, P., Koubský, P., et al. 2012, A\&A, 537, A59

Peters, G. J., \& Gies, D. R. 2002, in Exotic Stars as Challenges to Evolution, eds. C. A. Tout, \& W. Van Hamme, ASP Conf. Ser., 279, 149

Peters, G. J., Gies, D. R., Grundstrom, E. D., \& McSwain, M. V. 2008, ApJ, 686, 128

Peters, G. J., Pewett, T. D., Gies, D. R., et al. 2013, ApJ, 765, 2

Ruždjak, D. \& Božić, H., Harmanec, P., et al. 2009, A\&A, 506, 1319

Škoda, P. 1996, in Astronomical Data Analysis Software and Systems V, eds. G. H. Jacoby, \& J. Barnes, ASP Conf. Ser., 101, 187

Stellingwerf, R. F. 1978, AJ, 83, 118 